\title{
Diagnosis of large, mobile thrombus in transit within a patent foramen ovale by echocardiography
}

\author{
Jong-Ho Nam, Jong-Seon Park \\ Division of Cardiology, Department of Internal Medicine, Yeungnam University Medical Center, \\ Daegu, Republic of Korea
}

An 80-year-old woman was admitted for pulmonary embolism (PE; Fig. 1A), and deep vein thrombosis (DVT; Fig. 1B), and intravenous heparin was started. The next day, she was found to have right thumb motor weakness. Brain magnetic resonance imaging showed acute cerebral infarction of the left precentral gyrus, which is the hand motor area (Fig. 1C). Transthoracic echocardiography with contrast $\left(\right.$ Definity ${ }^{\circledR}$ ) showed large, mobile masses in atria without contrast enhancement, suggesting thrombi (Fig. 1D-F, Suppl. Video 1). Transesophageal echocardiography visualized a thrombus traversing through a patent foramen ovale (PFO) from right to left atrium (Fig. $1 \mathrm{G}$ ); agitated saline test demonstrated large right-to-left interatrial shunt (Fig. 1H). Surgical treatment was planned but follow-up echocardiography revealed that the thrombus size had decreased. Neurologic examination showed no neurologic deficit except previously observed right thumb motor weakness. After discharge and 3 months of follow-up under oral anticoagulant, there was no visible thrombus by transesophageal echocardiography. Considering paradoxical embolism and large right-to-left shunt, percutaneous transcatheter PFO closure was performed (Figulla Flex-II PFO Occluder ${ }^{\circledR}$; Fig. 1I).

In the setting of $\mathrm{PE}$ and elevated pulmonary arterial pressure, foramen ovale can become patent and trap a thrombus during its passage from the right to the left atrium. This thrombus in transit, impending paradoxical embolism, is rare and has a high mortality rate. Therefore, it should be suspected in patients who have unexplained systemic embolisms complicating DVT and PE. In such cases, echocardiography helps diagnose a rightto-left interatrial shunt and visualizes a thrombus in transit within a PFO.

\section{Acknowledgements}

This work was supported by 2020 Yeungnam University Research Grant.

Conflict of interest: None declared

Address for correspondence: Jong-Seon Park, MD, PhD, Division of Cardiology, Department of Internal Medicine, Yeungnam University Medical Center, 170 Hyeonchung-ro, Nam-gu, Daegu, 42415, Republic of Korea, tel: +82-53-620-3847, fax: +82-53-621-3310, e-mail: pjs@med.yu.ac.kr 


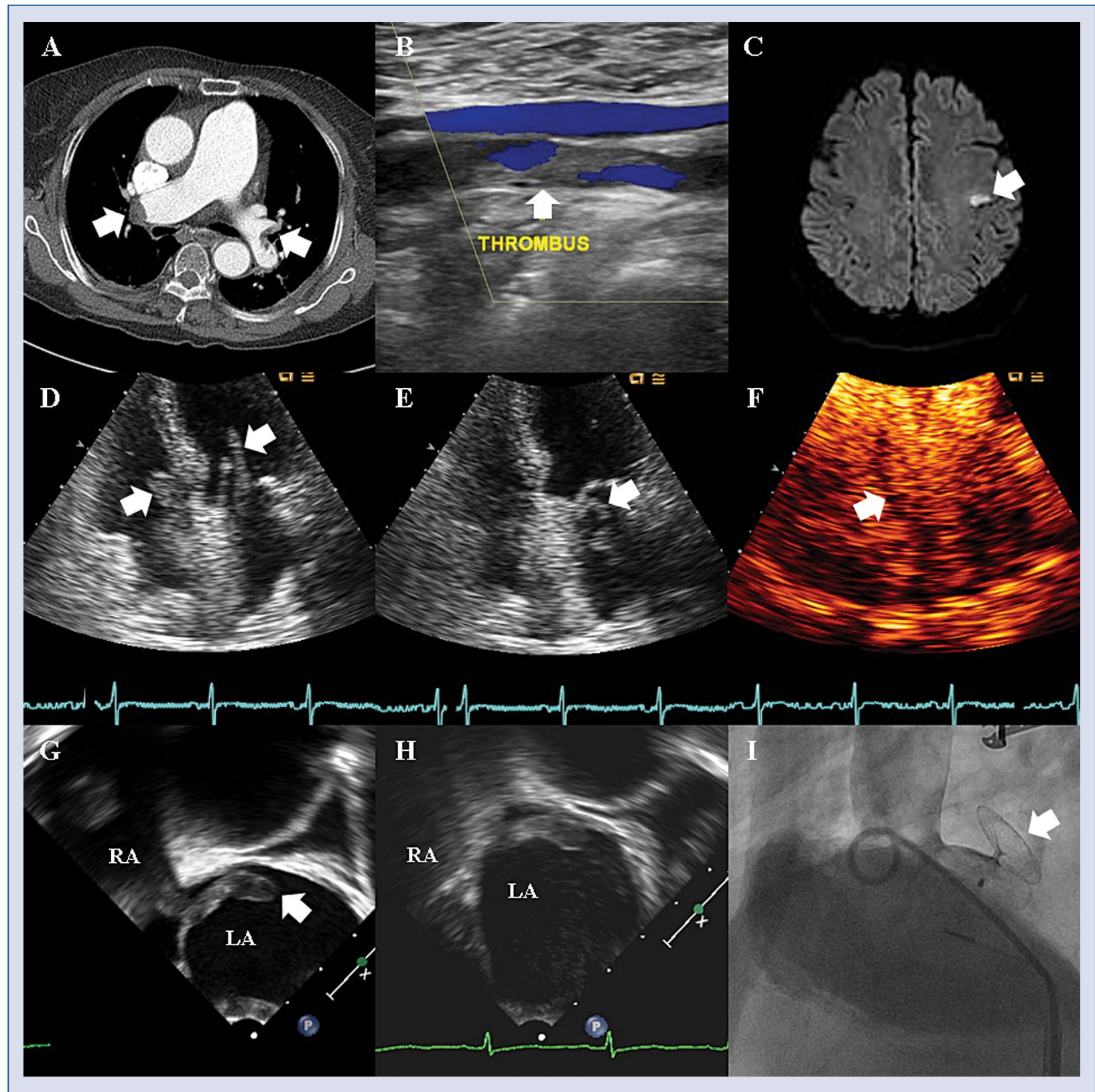

Figure 1. A. Chest computed tomography showing multiple filling defects (arrows) in both pulmonary arteries; B. Vascular duplex scan shows deep vein thrombosis (arrow) in the left popliteal vein; C. Brain magnetic resonance image showing acute cerebral infarction (arrow) of the left precentral gyrus; D-F. Apical 4-chamber transthoracic echocardiography shows large, mobile masses (arrows) in both atria at diastole (D) and systole (E), which were not enhanced by contrast (F); G. Mid-esophageal bicaval view of transesophageal echocardiography (TEE) shows a thrombus (arrow) in transit within the patent foramen ovale (PFO); H. Mid-esophageal bicaval view of TEE with agitated saline test confirming a large right-to-left shunt through the PFO; I. Fluoroscopy shows the transcatheter PFO occluder (arrow) placed successfully by intracardiac echocardiography and fluoroscopy; LA - left atrium; RA - right atrium. 